Europhysics Letters

PREPRINT

\title{
Entangling movable mirrors in a double-cavity system
}

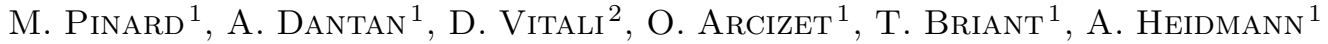 \\ 1 Laboratoire Kastler Brossel, Université Pierre et Marie Curie, Case 74, 4 place Jussieu, \\ 75252 Paris Cedex 05, France \\ 2 Dipartimento di Fisica, Università di Camerino, I-62032 Camerino, Italy
}

PACS. 42.50.Lc - Quantum fluctuations, quantum noise, and quantum jumps.

PACS. 03.67.Mn - Entanglement production, characterization and manipulation.

PACS. 05.40. Jc - Brownian motion.

\begin{abstract}
We propose a double-cavity set-up capable of generating a stationary entangled state of two movable mirrors at cryogenic temperatures. The scheme is based on the optimal transfer of squeezing of input optical fields to mechanical vibrational modes of the mirrors, realized by the radiation pressure of the intracavity light. We show that the presence of macroscopic entanglement can be demonstrated by an appropriate read out of the output light of the two cavities.
\end{abstract}

Introduction. - Quantum entanglement is a physical phenomenon in which the quantum states of two or more systems can only be described with reference to each other. This leads to correlations between observables of the systems that cannot be understood on the basis of local realistic theories $[1,2]$. Its importance today exceeds the realm of the foundations of quantum physics and entanglement has become an important physical resource that allows performing communication and computation tasks with an efficiency which is not achievable classically [3]. In particular, it is important to investigate under which conditions entanglement between macroscopic objects, each containing a large number of the constituents, can arise. Entanglement between two atomic ensembles has been successfully demonstrated in Ref. [4] by sending pulses of coherent light through two atomic vapor cells. Then, other proposals suggested to entangle a nano-mechanical oscillator with a Cooper-pair box [5], arrays of nanomechanical oscillators [6], two mirrors of an optical ring cavity [7], or two mirrors of two different cavities illuminated with entangled light beams [8]. Here we elaborate on these two latter schemes and propose a new double-cavity set-up able to generate a stationary entangled state of two vibrating cavity mirrors, exploiting the radiation pressure of the intracavity fields. Entanglement between mechanical degrees of freedom is achieved if the input fields are squeezed and if this squeezing is efficiently transferred to the movable mirrors. We show that a stationary entangled state can be generated with state-of-the-art apparata at cryogenic temperatures, and that it can be detected with a non-stationary homodyne measurement of the output light $[9,10]$.

(C) EDP Sciences 


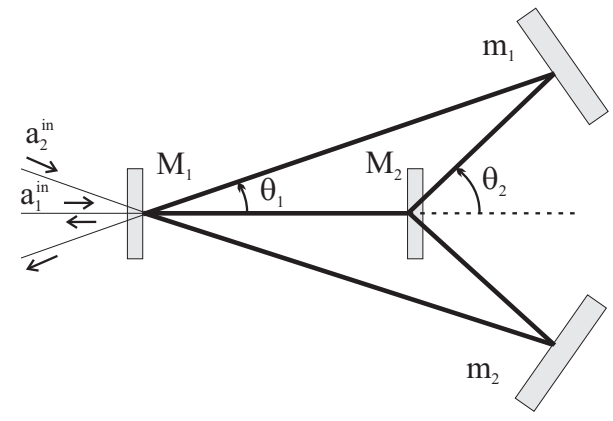

Fig. 1 - The double-cavity set-up. $M_{1}$ and $M_{2}$ are movable mirrors, while $m_{1}$ and $m_{2}$ are fixed. Fields $a_{1}, a_{2}$ are sent into the linear and folded cavities, respectively.

The system. - Let us consider two movable mirrors $M_{1}$ and $M_{2}$, oscillating at frequency $\Omega_{1}$ and $\Omega_{2}$ respectively, which interact with two field modes $a_{1}$ and $a_{2}$, with frequency $\omega_{L}$ (Fig. 11). Field $a_{1}$ is injected into a linear cavity constituted by mirrors $M_{1}$ and $M_{2}$, whereas $a_{2}$ is injected in a "folded" cavity formed by the movable mirrors $M_{1}$ and $M_{2}$, and by the fixed mirrors $m_{1}$ and $m_{2}$. Field $a_{1}$ is coupled to the length of the linear cavity, that is to the relative position of the mirrors, whereas field $a_{2}$ is coupled both to the center-of-mass and relative positions of the mirrors. We will see that for a judicious choice of the incidence angles $\theta_{1}$ and $\theta_{2}$, field $a_{2}$ is only coupled to the center of mass measured with respect to the positions of mirrors $m_{1}$ and $m_{2}$. It is then possible to decouple the relative and center-of-mass motions of the movable mirrors, as well as their coupling with the fields.

The motion of the mirrors generally corresponds to a superposition of many acoustic modes vibrations [11]. A single vibrational mode description can however be adopted whenever detection is limited to a frequency bandwidth including a single mechanical resonance. Assuming that mirrors $m_{1}$ and $m_{2}$ have much larger masses and no resonance frequency within the detection bandwidth, their motion can be neglected and the mechanical hamiltonian of the mirrors is given by

$$
H_{m}=\frac{P_{1}^{2}}{2 M_{1}}+\frac{P_{2}^{2}}{2 M_{2}}+\frac{1}{2} M_{1} \Omega_{1}^{2} Q_{1}^{2}+\frac{1}{2} M_{2} \Omega_{2}^{2} Q_{2}^{2} .
$$

We introduce the operators associated to the relative and the center of mass motions

$$
\begin{aligned}
Q_{c m} & =\frac{M_{1}}{M_{T}} Q_{1}+\frac{M_{2}}{M_{T}} Q_{2}, & & P_{c m}=P_{1}+P_{2}, \\
Q_{r} & =Q_{1}-Q_{2}, & & \frac{P_{r}}{\mu}=\frac{P_{1}}{M_{1}}-\frac{P_{2}}{M_{2}},
\end{aligned}
$$

where $M_{T}=M_{1}+M_{2}$ and $\mu=M_{1} M_{2} / M_{T}$ are the total and reduced masses of the system, respectively. If both mirrors have equal resonance frequencies $\Omega_{1}=\Omega_{2}=\Omega$, the mechanical Hamiltonian can be re-expressed as the sum of the Hamiltonians of two independent harmonic oscillators with frequency $\Omega$ and masses $\mu$ and $M_{T}$

$$
H_{m}=\frac{P_{c m}^{2}}{2 M_{T}}+\frac{1}{2} M_{T} \Omega^{2} Q_{c m}^{2}+\frac{P_{r}^{2}}{2 \mu}+\frac{1}{2} \mu \Omega^{2} Q_{r}^{2}
$$

The radiation pressure forces exerted by field $a_{1}$ on the mirrors are opposite, whereas they are in the same direction for field $a_{2}$. Taking into account the incident angles, the interaction 
Hamiltonians resulting from radiation pressure can be written as

$$
H_{1}=\frac{\hbar \omega_{c 1}}{L_{1}} a_{1}^{\dagger} a_{1}\left(Q_{1}-Q_{2}\right) \quad \text { and } \quad H_{2}=\frac{\hbar \omega_{c 2}}{L_{2}} a_{2}^{\dagger} a_{2}\left(Q_{1} \cos \theta_{1}+Q_{2} \cos \theta_{2}\right),
$$

where $L_{1}$ and $2 L_{2}$ are the lengths of the linear and folded cavities respectively, and $\omega_{c j}$ is the frequency of cavity mode $a_{j}$. The linear cavity mode $a_{1}$ is only coupled to the relative motion, while the folded cavity mode $a_{2}$ is coupled both to $Q_{r}$ and to $Q_{c m}$. One actually finds from Eqs. (23)

$$
H_{2}=\frac{\hbar \omega_{c 2}}{L_{2}} a_{2}^{\dagger} a_{2}\left(\cos \theta_{1}+\cos \theta_{2}\right) Q_{c m}+\frac{\hbar \omega_{c 2}}{L_{2}} a_{2}^{\dagger} a_{2}\left(-\frac{M_{1}}{M_{T}} \cos \theta_{1}+\frac{M_{2}}{M_{T}} \cos \theta_{2}\right) Q_{r} .
$$

If we choose the angles $\theta_{1}$ and $\theta_{2}$ so that

$$
\frac{M_{1}}{M_{2}}=\frac{\cos \theta_{1}}{\cos \theta_{2}}
$$

the second term in Eq. (6) vanishes and the radiation pressure couples $a_{2}$ to the center-of-mass motion only. We define the usual annihilation operators $b_{r}$ and $b_{c m}$ associated to the relative and center-of-mass motion respectively, as

$$
\begin{aligned}
& b_{r}=\frac{\mu \Omega Q_{r}+i P_{r}}{\sqrt{2 \hbar \mu \Omega}}=\frac{x_{r}+i p_{r}}{\sqrt{2}} \\
& b_{c m}=\frac{M_{T} \Omega Q_{c m}+i P_{c m}}{\sqrt{2 \hbar M_{T} \Omega}}=\frac{x_{c m}+i p_{c m}}{\sqrt{2}}
\end{aligned}
$$

where $x_{j}$ and $p_{j}$ satisfy $\left[x_{j}, p_{k}\right]=i \delta_{j k},(j, k=r, \mathrm{~cm})$. The radiation pressure interaction terms can then be rewritten as

$$
H_{1}=\hbar G_{1} a_{1}^{\dagger} a_{1}\left(b_{r}+b_{r}^{\dagger}\right) \quad \text { and } \quad H_{2}=\hbar G_{2} a_{2}^{\dagger} a_{2}\left(b_{c m}+b_{c m}^{\dagger}\right),
$$

where $G_{1}=\left(\omega_{c 1} / L_{1}\right) \sqrt{\hbar / 2 \mu \Omega}$ and $G_{2}=\left(\omega_{c 2} / L_{2}\right) \sqrt{\hbar / 2 M_{T} \Omega}\left(\cos \theta_{1}+\cos \theta_{2}\right)$ are the optomechanical coupling constants.

The Heisenberg-Langevin equations for the quantum-mechanical oscillators and the field modes which result from these couplings are, in the frame rotating at the laser frequency $\omega_{L}$,

$$
\begin{aligned}
& \dot{a}_{1}=-\left(\kappa_{1}+i \Delta_{1}\right) a_{1}-i G_{1} a_{1}\left(b_{r}+b_{r}^{\dagger}\right)+\sqrt{2 \kappa_{1}} a_{1}^{i n}, \\
& \dot{b}_{r}=-(\Gamma / 2+i \Omega) b_{r}+(\Gamma / 2) b_{r}^{\dagger}-i G_{1} a_{1}^{\dagger} a_{1}+\xi_{r}, \\
& \dot{a}_{2}=-\left(\kappa_{2}+i \Delta_{2}\right) a_{2}-i G_{2} a_{2}\left(b_{c m}+b_{c m}^{\dagger}\right)+\sqrt{2 \kappa_{2}} a_{2}^{i n}, \\
& \dot{b}_{c m}=-(\Gamma / 2+i \Omega) b_{c m}+(\Gamma / 2) b_{c m}^{\dagger}-i G_{2} a_{2}^{\dagger} a_{2}+\xi_{c m},
\end{aligned}
$$

where $\kappa_{j}$ are the cavity bandwidths, $\Delta_{j}=\omega_{c j}-\omega_{L}$ the cavity detunings, and $\Gamma$ the damping rate of the mirrors, assumed equal for both mirrors to ensure that the center-of-mass and the relative motions are not coupled via the dissipation process. We thus have two mechanical oscillators and two optical modes interacting in pairs: the relative motion only interacts with the linear cavity mode, and the center-of-mass with the folded cavity mode. The $\delta$-correlated noise operators $\xi$ 's are associated to the Brownian motion of the mirrors. They have zero-mean value and satisfy [12]

$$
\begin{aligned}
\left\langle\xi_{i}(t) \xi_{j}^{\dagger}\left(t^{\prime}\right)\right\rangle & =\Gamma\left(1+n_{T}\right) \delta\left(t-t^{\prime}\right) \delta_{i j} \\
\left\langle\xi_{i}^{\dagger}(t) \xi_{j}\left(t^{\prime}\right)\right\rangle & =\Gamma n_{T} \delta\left(t-t^{\prime}\right) \delta_{i j}
\end{aligned}
$$


$(i, j=c m, r)$, where $n_{T}=1 /\left(e^{\hbar \Omega / k_{B} T}-1\right)$ is the mean thermal phonon number at equilibrium temperature $T$. This corresponds to an Ohmic dissipation and the Markovian property is justified at not too low temperatures, i.e., $\hbar \Omega \ll k T$.

Steady state and fluctuations. - Setting the time-derivatives to zero in the previous equations yields the steady state values of the intracavity amplitudes and mirror positions

$$
\begin{aligned}
& E_{1}=\left\langle a_{1}\right\rangle=\frac{\sqrt{2 \kappa_{1}}\left\langle a_{1}^{i n}\right\rangle}{\kappa_{1}+i \Delta_{1}^{\prime}}, \quad \Delta_{1}^{\prime}=\Delta_{1}+G_{1}\left\langle b_{r}+b_{r}^{\dagger}\right\rangle, \quad\left\langle b_{r}\right\rangle=\frac{-G_{1}\left|E_{1}\right|^{2}}{\Omega}, \\
& E_{2}=\left\langle a_{2}\right\rangle=\frac{\sqrt{2 \kappa_{2}}\left\langle a_{2}^{i n}\right\rangle}{\kappa_{2}+i \Delta_{2}^{\prime}}, \quad \Delta_{2}^{\prime}=\Delta_{2}+G_{2}\left\langle b_{c m}+b_{c m}^{\dagger}\right\rangle, \quad\left\langle b_{c m}\right\rangle=\frac{-G_{2}\left|E_{2}\right|^{2}}{\Omega} .
\end{aligned}
$$

The effective detunings $\Delta_{j}^{\prime}$ include the mean displacements of the mirrors due to radiation pressure, which are proportional to the intracavity intensities $\left|E_{j}\right|^{2}$. We can arbitrarily choose the detunings $\Delta_{j}^{\prime}$ by setting the detunings of the cavities, as long as we stay in the stable domain of the bistability induced by the optomechanical coupling. We consider in the following the case of detuned cavities with $\Delta_{1}^{\prime}=\Delta_{2}^{\prime}=\Omega$.

Eqs. (11.12) and (13 14) are decoupled and formally identical: we first treat the case of the relative motion. Linearizing Eqs. (11.12) around the steady state [13], the fluctuations of operators $a_{1}$ and $b_{r}$ obey the following equations

$$
\begin{aligned}
& \delta \dot{a}_{1}=-\left(\kappa_{1}+i \Delta_{1}^{\prime}\right) \delta a_{1}-i G_{1} E_{1}\left(\delta b_{r}+\delta b_{r}^{\dagger}\right)+\sqrt{2 \kappa_{1}} \delta a_{1}^{i n}, \\
& \delta \dot{b}_{r}=-(\Gamma / 2+i \Omega) \delta b_{r}+(\Gamma / 2) \delta b_{r}^{\dagger}-i G_{1}\left(E_{1}^{*} \delta a_{1}+E_{1} \delta a_{1}^{\dagger}\right)+\xi_{r} .
\end{aligned}
$$

We assume $\Omega \gg \kappa_{j}$, which means that the cavities are strongly off-resonant from the fields. Moving to the frame rotating at frequency $\Omega$ and neglecting the fast rotating terms, one gets

$$
\begin{aligned}
& \delta \dot{\tilde{a}}_{1}=-\kappa_{1} \delta \tilde{a}_{1}-G_{1}\left|E_{1}\right| \delta \tilde{b}_{r}+\sqrt{2 \kappa_{1}} \delta \tilde{a}_{1}^{i n}, \\
& \delta \dot{\tilde{b}}_{r}=-(\Gamma / 2) \delta \tilde{b}_{r}+G_{1}\left|E_{1}\right| \delta \tilde{a}_{1}+\tilde{\xi}_{r},
\end{aligned}
$$

where the slow observables in the rotating frame are given by $\tilde{o}(t)=o(t) e^{i \Omega t}$ and we have chosen by convention the phases of the input fields to be real (so that $E_{j}=-i\left|E_{j}\right|$ ). The interesting regime for quantum state transfer is when the fields adiabatically follow the mirrors, which is the case for mirrors with high-Q mechanical factors $\left(\Gamma, G_{j}\left|E_{j}\right| \ll \kappa_{j}\right)[14]$. The mirror dynamics then reduce to

$$
\delta \dot{\tilde{b}}_{r}=-\tilde{\gamma}_{1} \delta \tilde{b}_{r}+\sqrt{\Gamma_{c 1}} \delta \tilde{a}_{1}^{i n}+\tilde{\xi}_{r}
$$

where $\Gamma_{c 1}=2 G_{1}^{2}\left|E_{1}\right|^{2} / \kappa_{1}$ represents the effective relaxation rate induced by radiation pressure [15] and $\tilde{\gamma}_{1}=\left(\Gamma+\Gamma_{c 1}\right) / 2$. A similar equation can be obtained from Eqs. (13 14) for the centerof-mass operator $b_{c m}$

$$
\delta \dot{\tilde{b}}_{c m}=-\tilde{\gamma}_{2} \delta \tilde{b}_{c m}+\sqrt{\Gamma_{c 2}} \delta \tilde{a}_{2}^{i n}+\tilde{\xi}_{c m}
$$

Noise spectrum and entanglement. - Since $\left[\tilde{x}_{r}, \tilde{p}_{c m}\right]=0$, the relative position and the center-of-mass momentum of the two movable mirrors play the role of EPR operators [1]: their variances can be simultaneously zero, and if they are small enough, the corresponding state possesses nonlocal correlations. To be more specific, according to the inseparability sufficient criterion of [16], the steady state of the two movable mirrors is entangled if

$$
\Delta x_{r}^{2}+\Delta p_{c m}^{2}<2 \sqrt{\frac{\mu}{M_{T}}},
$$


where $\Delta o^{2}$ denotes the stationary variance of $o$. For instance, in the case of similar mirrors $\left(M_{1} \simeq M_{2}\right)$, this criterion is satisfied if the sum of the EPR variances is less than 1 .

Assuming for simplicity $\Gamma_{c 1}=\Gamma_{c 2}=\Gamma_{c}$, the Fourier transforms of Eqs. (19, 20) yield

$$
\delta \tilde{b}_{r}(\omega)=\frac{\sqrt{\Gamma_{c}} \delta \tilde{a}_{1}^{i n}(\omega)+\tilde{\xi}_{r}(\omega)}{\tilde{\gamma}-i \omega}, \quad \delta \tilde{b}_{c m}(\omega)=\frac{\sqrt{\Gamma_{c}} \delta \tilde{a}_{2}^{i n}(\omega)+\tilde{\xi}_{c m}(\omega)}{\tilde{\gamma}-i \omega}
$$

with $\tilde{\gamma}=\tilde{\gamma}_{1}=\tilde{\gamma}_{2}$. The widths of the spectra are broadened from $\Gamma / 2$ to $\left(\Gamma+\Gamma_{c}\right) / 2$ by radiation pressure effects. The Brownian motion characterized by the operators $\tilde{\xi}_{r}$, $\tilde{\xi}_{c m}$ is reduced by a self-cooling effect for large $\Gamma_{c}[15]$. On the other hand, the quantum fluctuations of $a_{1}^{i n}\left(a_{2}^{i n}\right)$ also imprint on $b_{r}\left(b_{c m}\right)$ when $\Gamma_{c} \gg \Gamma$. Assuming that fields $a_{1}^{i n}$ and $a_{2}^{i n}$ are respectively amplitude- and phase-squeezed around $\Omega$ with a bandwidth larger than $\Gamma+\Gamma_{c}$, the amplitude fluctuations of $a_{1}^{i n}$ are transferred to $b_{r}$, whereas the phase fluctuations of $a_{2}^{i n}$ are transferred to $p_{c m}$. The EPR variances are then

$$
\begin{aligned}
\Delta x_{r}^{2} & =\frac{1}{2}\left[\frac{\Gamma_{c}}{\Gamma+\Gamma_{c}} e^{-2 r_{1}}+\frac{\Gamma}{\Gamma+\Gamma_{c}}\left(1+2 n_{T}\right)\right], \\
\Delta p_{c m}^{2} & =\frac{1}{2}\left[\frac{\Gamma_{c}}{\Gamma+\Gamma_{c}} e^{-2 r_{2}}+\frac{\Gamma}{\Gamma+\Gamma_{c}}\left(1+2 n_{T}\right)\right],
\end{aligned}
$$

where $\Delta^{2} X_{1}^{i n}=e^{-2 r_{1}}, \Delta^{2} Y_{2}^{i n}=e^{-2 r_{2}}$ are the variances of the amplitude and phase quadratures, respectively $\left[X=a+a^{\dagger}\right.$ and $\left.Y=i\left(a^{\dagger}-a\right)\right]$. These equations stress the physical processes able to produce the stationary entanglement: first, the mirrors thermal noise is reduced by self-cooling when $\Gamma_{c} \gg 2 \Gamma n_{T}$. Secondly, the quantum fluctuations of the input fields are transferred to the center-of-mass and relative motions of the two mirrors with an optimal efficiency at the resonance condition $\Delta_{1}^{\prime}=\Delta_{2}^{\prime}=\Omega$. This process is very similar to the quantum state transfer from light to atomic variables $[9,10]$.

One gets for coherent inputs $\left(r_{1}=r_{2}=0\right)$

$$
\Delta x_{r}^{2}=\Delta p_{c m}^{2}=\frac{1}{2}+\frac{\Gamma}{\Gamma+\Gamma_{c}} n_{T}
$$

The relative position and the center-of-mass momentum can never be squeezed, and therefore the two mirrors are never entangled. When the incident fields are squeezed, it is clear that the mirror motions reproduce the squeezed fluctuations of the incident fields for a large enough cooling rate $\Gamma_{c} / \Gamma \gg 2 n_{T}$. At a given temperature, this condition can always be achieved for large enough intracavity intensities. For almost identical masses $\left(M_{1} \simeq M_{2}\right)$, both mirrors are then in an entangled state:

$$
\Delta x_{r}^{2}+\Delta p_{c m}^{2} \simeq \frac{1}{2}\left(e^{-2 r_{1}}+e^{-2 r_{2}}\right)<1
$$

For perfectly squeezed inputs, this corresponds to a realization of the original EPR paradox for the mechanical oscillators, with perfect position correlations and momentum anti-correlations.

Such a stationary entanglement can already be achieved with state-of-the-art apparata, working at cryogenic temperatures, as shown in Fig. 2. where the (normalized) criterion (21) is plotted versus the mirror temperature. The first curve refers to two identical mirrors with $M_{1} \simeq M_{2}=1 \mathrm{mg}, \Omega / 2 \pi=1 \mathrm{MHz}, \Gamma=1 \mathrm{~Hz}$, cavities with finesse $10^{5}$, lengths $L_{1}=3 \mathrm{~cm}$, $L_{2}=9 \mathrm{~cm}$, input squeezing such that $r_{1}=r_{2}=2$, and input powers $P_{1}^{\text {in }}=30 \mathrm{~mW}, P_{2}^{i n}=1.2$ $\mathrm{W}\left(\Gamma_{c} / \Gamma=10^{3}\right)$. The second curve corresponds to two micro-electro mechanical mirrors (MEMS) with masses $1 \mu \mathrm{g}$, for which much smaller input powers $P_{1}^{\text {in }}=0.3 \mathrm{~mW}, P_{2}^{\text {in }}=12$ $\mathrm{mW}\left(\Gamma_{c} / \Gamma=10^{4}\right)$ and not as low temperatures are required. Thanks to the self-cooling process the quantum state transfer is efficient even at relatively high temperatures. 
EUROPHYSICS LETTERS

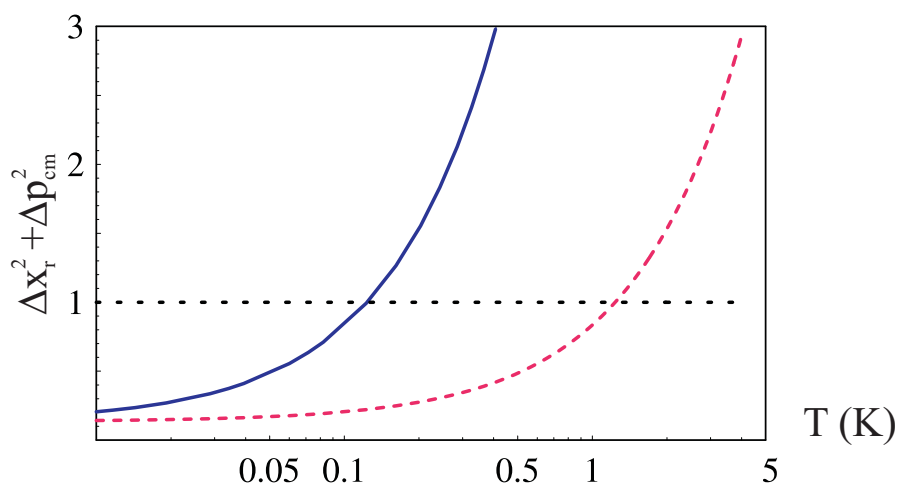

Fig. 2 - Plots of the normalized sum of variances $\Delta x_{r}^{2}+\Delta p_{c m}^{2}$ versus temperature, in the case of almost identical mirrors $M_{1} \simeq M_{2}=1 \mathrm{mg}$ (full line), $M_{1} \simeq M_{2}=1 \mu \mathrm{g}$ (dashed line). The dotted horizontal line denotes the region below which the stationary state of the two movable mirrors is entangled. See text for the parameter values.

Readout. - The motion of a movable mirror is usually measured by monitoring the phase of the field reflected by a high-finesse resonant cavity $[7,17]$. In this paper, we adopt another strategy to readout the quantum noise of the mirrors, inspired by optical readout of atomic ensemble quantum states $[9,10]$. Let us assume that after completion of the fluctuation transfer between the fields and the mirrors, one rapidly switches off the squeezings entering the cavities, the field intensities being kept constant. We therefore start in a regime in which the thermal noise has been damped by the self cooling process and we want to read out the mirror squeezed fluctuations of the fields exiting the cavities: $a_{j}^{\text {out }}=\sqrt{2 \kappa_{j}} a_{j}-a_{j}^{\text {in }}$.

Denoting by $t=0$ this switching time, one gets after integrating (19)

$$
\delta \tilde{a}_{1}^{o u t}(t)=\delta \tilde{a}_{1}^{i n}(t)-\Gamma_{c} \int_{0}^{t} d t^{\prime} e^{-\tilde{\gamma}\left(t-t^{\prime}\right)} \delta \tilde{a}_{1}^{i n}\left(t^{\prime}\right)-\sqrt{\Gamma_{c}} \delta \tilde{b}_{r}(0) e^{-\tilde{\gamma} t}-\sqrt{\Gamma_{c}} \int_{0}^{t} d t^{\prime} e^{-\tilde{\gamma}\left(t-t^{\prime}\right)} \tilde{\xi}_{r}\left(t^{\prime}\right) .
$$

The term proportional to $\delta \tilde{b}_{r}(0)$ carries the information to be measured. The two-time correlation function of the outgoing amplitude quadrature is then given by

$$
\left\langle\delta \tilde{X}_{1}^{\text {out }}(t) \delta \tilde{X}_{1}^{\text {out }}\left(t^{\prime}\right)\right\rangle=\delta\left(t-t^{\prime}\right)+\frac{n_{T} \Gamma \Gamma_{c}}{\tilde{\gamma}} e^{-\tilde{\gamma}\left|t-t^{\prime}\right|}+2 \Gamma_{c}\left[\Delta x_{r}^{2}(0)-\frac{\Gamma_{c}+\Gamma\left(1+2 n_{T}\right)}{2\left(\Gamma+\Gamma_{c}\right)}\right] e^{-\tilde{\gamma}\left(t+t^{\prime}\right)} .
$$

The two first terms give the $\delta$-correlated function of the field in the absence of coupling and the contribution of the mirror thermal noise, respectively. The last term is proportional to the difference between the initial variance $\Delta x_{r}^{2}(0)$ and the final one $\Delta x_{r}^{2}(\infty)$, corresponding to the thermal equilibrium in the self-cooled regime (see Eq. (23) with $r_{1}=0$ ).

In order to efficiently measure the stored squeezing, we perform a homodyne detection of the outgoing field fluctuations using a local oscillator with a temporal profile matching that of the mirror response: $E(\tau) \propto e^{-\tilde{\gamma} \tau}$. We then measure the noise starting at a given time $t$ and integrating over a time $t_{m}$ assumed large with respect to $\tilde{\gamma}^{-1}$. The noise spectrum is integrated around frequency $\Omega$ with a frequency bandwidth $\Delta \omega=2 \pi / t_{m}$. The resulting normalized noise power is equal to

$$
P_{1}(t)=\frac{1}{I(t)} \int_{-\Delta \omega / 2}^{\Delta \omega / 2} \frac{d \omega}{2 \pi} \int_{t}^{t+t_{m}} d \tau \int_{t}^{t+t_{m}} d \tau^{\prime} E(\tau) E^{*}\left(\tau^{\prime}\right) e^{-i \omega\left(\tau-\tau^{\prime}\right)}\left\langle\delta \tilde{X}_{1}^{\text {out }}(\tau) \delta \tilde{X}_{1}^{\text {out }}\left(\tau^{\prime}\right)\right\rangle,
$$


where $I(t)=E(t) E^{*}(t)$. From Eq. (27) this noise power can be written as the sum of constant noise terms and a signal term depending on the initial time $t$,

$$
P_{1}(t)=\frac{1}{2 \tilde{\gamma} t_{m}}\left[1+\frac{4 \Gamma \Gamma_{c}}{\left(\Gamma+\Gamma_{c}\right)^{2}} n_{T}+\frac{2 \Gamma_{c}}{\Gamma+\Gamma_{c}}\left[\Delta x_{r}^{2}(0)-\Delta x_{r}^{2}(\infty)\right] e^{-2 \tilde{\gamma} t}\right]
$$

The first two terms represent the field shot-noise level and the contribution of the mirror thermal noise, whereas the signal term is proportional to the difference between the mirror squeezed initial variance and its variance at thermal equilibrium. If the self-cooling is strong enough to reduce the thermal noise $\left(\Gamma_{c} / \Gamma \gg 1,2 n_{T}\right)$, the noise terms reduce to 1 and $\Delta x_{r}^{2}(\infty) \sim 1 / 2$, so that one effectively measures squeezing in the outgoing field, leaking out of the cavity in a time $\tilde{\gamma}^{-1}$ and directly related to the squeezing $\left(1 / 2-\Delta x_{r}^{2}(0)\right)$ initially stored

in the mirrors. Of course, measuring the fluctuations of $\tilde{Y}_{2}^{\text {out }}$ would yield a similar measurement of $\Delta p_{c m}^{2}(0)$. This readout technique thus provides an unambiguous and experimentally accessible evidence that the mirrors were entangled. Indeed, if one were to use motionless mirrors for instance, the squeezing exiting the cavity would disappear in a time $t \sim \kappa^{-1}$ much shorter than $\tilde{\gamma}^{-1}$.

Conclusion. - We have proposed a new scheme for generating and detecting a stationary entangled state of two vibrational modes of a pair of mirrors in a double-cavity system. Entanglement is achievable at few Kelvin degrees if the input light is appropriately squeezed and for sufficiently large intracavity light intensities and small mirrors (MEMS).

This work was partially supported by the COVAQIAL European Project No. FP6-511004. Laboratoire Kastler Brossel is an Unité Mixte du Centre National de la Recherche Scientifique, de l'Ecole Normale Supérieure et de l'Université Pierre et Marie Curie.

\section{REFERENCES}

[1] Einstein A., Podolsky B. and Rosen R., Phys. Rev., 47 (1935) 777.

[2] Bell J.S., Physics (NY), 1 (1964) 195.

[3] Nielsen M.A. and Chuang I.L., Quantum Computation and Quantum Information (Cambridge University Press, Cambridge) 2000.

[4] JulsgaArd B. et al., Nature (London), 413 (2001) 400.

[5] Armour A.D., Blencowe M.P. and Schwab K.C., Phys. Rev. Lett., 88 (2002) 148301.

[6] Eisert J., Plenio M.B., Bose S., Hartley J., Phys. Rev. Lett., 93 (2004) 190402.

[7] Mancini S., Giovannetti V., Vitali D. and Tombesi P., Phys. Rev. Lett., 88 (2002) 120401.

[8] Zhang J., Peng K. and Braunstein S.L., Phys. Rev. A, 68 (2003) 013808.

[9] Dantan A. and Pinard M., Phys. Rev. A, 69 (2004) 043810.

[10] Dantan A., Bramati A. and Pinard M., Europhys. Lett., 67 (2004) 881.

[11] Pinard M., Hadjar Y. and Heidmann A., Eur. Phys. J. D, 7 (1999) 107.

[12] Gardiner C.W. and Zoller P., Quantum Noise (Springer, Berlin) 2000, p. 71.

[13] Fabre C., Pinard M., Bourzeix S., Heidmann A., Giacobino E. and Reynaud S., Phys. Rev. A, 49 (1994) 1337; Mancini S. and Tombesi P., Phys. Rev. A, 49 (1994) 4055.

[14] Briant T., Cohadon P.F., Heidmann A. and Pinard P., Phys. Rev. A, 68 (2003) 033823.

[15] Braginsky V.B. and Vyatchanin S.P., Phys. Lett. A, 293 (2002) 228; Metzger C.H. and Karrai K., Nature (London), 432 (2004) 1002.

[16] Giovannetti V., Mancini S., Vitali D. and Tombesi P., Phys. Rev. A, 67 (2003) 022320.

[17] Hadjar Y. et al., Europhys. Lett., 47 (1999) 545; Cohadon P.F., Heidmann A. and Pinard M., Phys. Rev. Lett., 83 (1999) 3174. 